\title{
Optimality conditions for reflecting boundary control problems
}

\author{
Oana Silvia Serea
}

\begin{abstract}
We consider a control problem with reflecting boundary and obtain necessary optimality conditions in the form of the maximum Pontryagin principle. To derive these results we transform the constrained problem in an unconstrained one or we use penalization techniques of Morreau-Yosida type to approach the original problem by a sequence of optimal control problems with Lipschitz dynamics. Then nonsmooth analysis theory is used to study the convergence of the penalization in order to obtain optimality conditions.
\end{abstract}

Mathematics Subject Classification. 34K35, 49J24, 49L20, 49L25, 93B18, 93C15.

Keywords. Boundary reflection, Pontryagin's principle, Necessary conditions, Dynamic programming, Synthesis for optimal solutions.

\section{Introduction}

Consider the following differential variational inequality:

$$
\left\{\begin{array}{l}
i) y^{\prime}(t) \in f(y(t), u(t))-N_{K}(y(t)) \quad \text { a.e } t \in[0, T] \\
i i) y(t) \in K \quad \text { for all } t \in[0, T], y(0)=x
\end{array}\right.
$$

where $u(\cdot):[0, \infty) \rightarrow U$ is a measurable function and $T \geq 0$ is fixed. Here $K$ is a nonempty closed subset of $\mathbb{R}^{N}, U$ is a compact metric space, $f$ is a bounded function from $\mathbb{R}^{N} \times U$ into $\mathbb{R}^{N}$ and $N_{K}(x)$ is the normal cone to $K$ at $x \in K$. We notice that $N_{K}(x)=\{0\}$ whenever $x \in \stackrel{\circ}{K} ; f$ is only modified on the boundary of $K$, such that (1) is a problem with reflection at the boundary (see [31]).

We denote by $\mathcal{U}$ the set of measurable controls on $[0, T]$ with values in $U$. Let $g: \mathbb{R}^{N} \rightarrow \mathbb{R}$ be a continuous function and consider the Mayer's problem defined by:

$$
\text { minimize }\left\{g\left(y^{u}(T)\right) \mid u(\cdot) \in \mathcal{U} \text { and } y^{u}(\cdot) \text { satisfies }(1)\right\},
$$

where $y^{u}(\cdot)$ denotes the solution of (1) with the initial condition $y^{u}(0)=x$. 
Note that, the set $K$ is viable for the dynamics of the reflected control system. When $K$ is regular enough, the viability property, guarantees that all the trajectories of this controlled system stay in $K$ for ever $[1,13,31]$. Therefore, the state condition $y(t) \in K$ is not a severe constraint for the optimization problem. However, the presence of the reflection on the boundary of $K$ implies some difficulties for getting necessary optimality conditions for the problem $(\mathcal{P})$. Indeed, the derivative of the normal cone, which is linked with the second order normal cone is not very regular in general (see [30] for details). Consequently, it is not very easy to handle the problem directly. Nevertheless, we succeed to deal with this problem by using an approximating "from exterior" method or by transforming the constrained problem in an unconstrained one. This approximation is possible if one assumes some regularity on the reflection set. This is the case whenever the reflection set is a proximal retract (see Definition 2.1 and the comments following it). Moreover, when the set $K$ is convex and smooth we provide a way to calculate the adjoint state for the reflected problem. Let us briefly present the main idea of our method. Consider the following approximating control systems (for $n \geq 1$ ):

$$
y^{\prime}(t)=f(y(t), u(t))-\nabla q_{n}(y(t)) \quad \text { for a.e } t \in[0, T],
$$

where the function $q: \mathbb{R}^{N} \rightarrow \mathbb{R}_{+}$is given by

$$
q(y):=d_{K}(y) \text { for all } y \in \mathbb{R}^{N}
$$

and $q_{n}: \mathbb{R}^{N} \rightarrow \mathbb{R}_{+}$is it's Moreau Yosida regularization:

$$
q_{n}(y):=\inf _{z \in \mathbb{R}^{N}}\left\{q(z)+\frac{n}{2}\|z-y\|^{2}\right\} \text { for all } y \in \mathbb{R}^{N} .
$$

Using the regularity properties of the function $q_{n}$ we can prove that every admissible trajectory solution of (1) can be approximated by a sequence $\left(y_{n}^{u_{n}}(\cdot)\right)_{n}$, where for every $n,\left(y_{n}^{u_{n}}\right)(\cdot)$ is solution to $(2)$ with the initial condition $\left(y_{n}^{u_{n}}\right)(0)=x$, so that the control problem $(\mathcal{P})$ can be also approximated by the following sequence of control problems (for $n \geq 1$ ):

$$
\text { minimize }\left\{g\left(y_{n}^{u}(T)\right) \mid u(\cdot) \in \mathcal{U} \text { and } y_{n}^{u}(\cdot) \text { satisfies }(2)\right\} \text {. }
$$

Obviously, the reflected control problem $(\mathcal{P})$ can be seen as a state-constrained control problem of differential inclusion systems. The reflected dynamics has the advantage that for every measurable control $u(\cdot)$ there exists a solution in $K$ because of the viability property. So, in this case the set of "admissible controls" coincide with $\mathcal{U}$ which is very advantageous for obtaining optimal trajectories i. e. solutions for the minimizing problem $(\mathcal{P})$. Let us recall that necessary conditions for state-constrained control problems governed by ordinary differential equations have been studied by many authors since the early sixties (see for instance $[9,11,12,16,24,25,33]$ and references therein). These necessary conditions are given in the form of a maximum principle. We refer also to $[15,18,19,29]$ for constrained problems governed by differential inclusions in the form of $\dot{y}(t) \in F(t, y(t))$, with a Lipschitz setvalued map $F$. In this framework, necessary optimality conditions have been 
derived in [19]. These conditions use a linearization of $F$ by closed convex processes, which are Lipschitz with respect to the state. In our case we need to suppose that the set $K$ is convex and we use an approximation via the distance function [see (2)] in order to obtain the main result.

Equation (1) modelize several important applications in mechanics. Indeed it is used to the study of the concept of a standard inelastic shock introduced in [26]. Moreover, numerical methods are developed in [10] for the study of systems composed of interacting rigid bodies. One of the methods uses the Moreau's sweeping process, which is similar to a reflected problem. Finally, let us mention that some optimal control problems governed by variational inequalities in infinite dimension spaces have been also studied in several works. Let us refer to $[7,8]$ and the references therein. In these works, the set $K$ is supposed to be a half space.

This paper is organized as follows. In Sect. 2, we present the optimal control problem and the assumptions that will be considered. Section 3 is devoted to the main results of the paper. We continue with the study of the approximating systems in Sect. 4. In the last section we complete the proof of the results presented in Sect. 3 .

Notations. In this paper, we denote by $d_{A}(x):=\inf _{y \in A}\|x-y\|$ the distance function to a set $A \subset \mathbb{R}^{N} ;\|\cdot\|$ and $\langle\cdot\rangle$ are the Euclidian norm and scalar product in $\mathbb{R}^{N}$, respectively. We also denote by $|\cdot|_{\infty}$ the infinity norm in $\mathbb{R}^{N}$. Moreover, $B$ denotes the closed unit sphere of $\mathbb{R}^{N}$. For any $X \subset \mathbb{R}^{N}$ we denote by $\bar{X}$ its closure, by $\stackrel{\circ}{X}$ its interior, and by $\overline{c o}(X)$ the closed convex hull of $X$.

\section{Setting of the problem}

\subsection{Assumptions}

Let $K$ be a closed subset of $\mathbb{R}^{N}$. We recall that the normal cone to the set $K$ at $x \in K$ is a convex set defined by:

$$
N_{K}(x)=\left\{v \in \mathbb{R}^{N} \mid\langle v, y-x\rangle \leq o(\|y-x\|) \text { for all } y \in K, y \rightarrow x\right\} .
$$

Let $f: \mathbb{R}^{N} \times U \rightarrow \mathbb{R}^{N}$ be a continuous function. For any measurable function $u(\cdot):[0, \infty) \rightarrow U$ we consider the associated trajectory satisfying:

$$
\left\{\begin{array}{l}
\text { i) } y^{\prime}(t) \in f(y(t), u(t))-N_{K}(y(t)) \quad \text { for a.e } t \in[0, T] \\
i i) y(t) \in K \quad \text { for all } t \in[0, T]
\end{array}\right.
$$

with $y(0)=x$. We consider the following assumption:

[A1] $f: \mathbb{R}^{N} \times U \rightarrow \mathbb{R}$ is of class $C^{1}$ and satisfies:

$$
\left\{\begin{array}{l}
\|f(x, u)-f(y, u)\| \leq L_{f}\|x-y\|, \\
\text { the set } f(x, U) \text { is convex, for all } x, y \in \mathbb{R}^{N} \text { and } u \in U,
\end{array}\right.
$$

where $L_{f}$ is a positive constant.

For the study of control systems with reflecting boundary, we suppose that $K$ is a proximal retract subset in $\mathbb{R}^{n}$ i. e.: 
Definition 2.1. A closed set $K \subset \mathbb{R}^{N}$ is called proximal retract if there exists a neighborhood $I$ of $K$ such that the projection $\Pi_{K}(\cdot)$ is single-valued in $I$, with $\Pi_{K}(x):=\left\{z \in K \mid\|x-z\|=\inf _{y \in K}\|x-y\|\right\}$ for all $x \in \mathbb{R}$.

The class of proximal retracts includes closed, convex subsets of $\mathbb{R}^{N}$ and subsets of $\mathbb{R}^{N}$ of class $C^{1,1}$. Another class of proximal retracts is the class of weakly convex sets (see [17] for the definition and the geometrical interpretation). A complete characterization of proximal retract sets can be found in [28, Theorem 4.1, p. 5245]. In particular, such sets have the property that there exists a $\rho>0$ such that the set $K$ has the exterior sphere property with radius $\rho$ :

Definition 2.2. We say that $K$ satisfies an exterior sphere condition of radius $\rho>0$ at a point $x \in \partial K$ if $x$ belongs to some closed ball centered in $z$, such that $z+\rho \bar{B} \subset \mathbb{R}^{N} \backslash \stackrel{\circ}{K}$. We say that $K$ satisfies an exterior sphere condition of radius $\rho$, if $K$ satisfies an exterior sphere condition of radius $\rho$ at every $x \in \partial K$.

\subsection{Control systems with reflecting boundary}

A detailed study of the differential inequality (1) is presented in [31]. For the reader's convenience, we recall some properties of the set of solutions to this controlled system.

First, consider a set-valued map $F: \mathbb{R}^{N} \rightsquigarrow \mathbb{R}^{N}$, and the following differential inclusion:

$$
\left\{\begin{array}{l}
\text { i) } z^{\prime}(t) \in F(z(t))-N_{K}(z(t)) \quad \text { for a.e } t \in[0, T], \\
i i) z(t) \in K, \quad \text { for all } t \in[0, T], z(0)=x
\end{array}\right.
$$

In the sequel, we denote by $S_{F-N_{K}}(x)$ the set of solutions to (5) starting from $x \in K$. We consider that $S_{F-N_{K}}(y)=\emptyset$ for every $y \in K^{c}$, because $N_{K}(y)$ is not defined. By using [1, Theorem 10.1.1], we can prove the following results (see $[13,26,31,32])$ :

Proposition 2.3. Suppose that $F$ is upper semicontinuous (usc, for short) with non-empty compact convex values, has a linear growth, and $K$ is a proximal retract set. Then

(i) For every $x \in K, E q$. (5) admits an absolutely continuous solution $z(\cdot)$.

(ii) The restriction of the map $x \rightsquigarrow S_{F-N_{K}}(x)$ to $K_{1}$ is compact into $K_{1} \times$ $W^{1,1}(0, \infty ; K) e^{-b t}$ for every $K_{1}$ compact subset of $K$ and for all $b>L_{f}$, where $L_{f}$ is the Lipschitz constant in (A1).

From now on, we consider the set-valued map $F$ given by the equality

$$
F(y)=f(y, U)=\{f(y, u), u \in U\}, \quad \text { for all } y \in \mathbb{R}^{N} .
$$

Under assumption (4), we can check that $F$ is usc, has a linear growth, and has nonempty compact convex values. Moreover, let $K$ be a proximal retract set. Then we employ results concerning the existence of measurable selections from (see Chapter 2 in [1]). Consequently, we obtain that, for each solution $y(\cdot)$ to the differential inclusion (5), starting at $x$, there exists $u(\cdot) \in \mathcal{U}$ 
such that $y(\cdot)$ is equal to $y^{u}(\cdot)$, which is the solution of (1). A proof of this claim can be found in [31]. Therefore, we have:

$$
S_{F-N_{K}}(x)=\mathcal{S}(x) \quad \text { for all } x \text { in } K,
$$

where $\mathcal{S}(x)$ is the set of absolutely continuous solutions to (1). On other hand, by straightforward computation using the fact that $K$ is a proximal retract and Gronwall's inequality, we obtain the following estimation:

Lemma 2.4. Assume that (4) holds true. Let $K$ be a proximal retract set, $y_{1}^{u}(\cdot) \in \S\left(x_{1}\right), y_{2}^{u}(\cdot) \in \S\left(x_{2}\right)$ with $x_{1}, x_{2}$ in $K$ and fixed $u(\cdot) \in \mathcal{U}$. Then, for every $T>0$, there exist a constant $C>0$ depending on $T$ such that:

$$
\sup _{t \in[0, T]}\left\|y_{1}^{u}(t)-y_{2}^{u}(t)\right\| \leq C\left\|x_{1}-x_{2}\right\| \text {. }
$$

As a direct consequence of the above estimation we obtain:

Corollary 2.5. Assume that (4) holds true and $K$ is a proximal retract set. Then there exists an unique solution of (1) in $K$ for every fixed $u(\cdot) \in \mathcal{U}$.

Let $g$ be a function from $\mathbb{R}^{N}$ to $\mathbb{R}$. We assume that:

(A2) $g$ is of class $C^{1}$ on $\mathbb{R}^{N}$.

We consider the control problem:

$$
\text { minimize }\left\{g\left(y^{u}(T)\right) \mid u \in \mathcal{U}, y^{u}(\cdot) \text { solves (1) and } y^{u}(0)=x\right\} \text {. }
$$

The value function associated to this control problem is defined by:

$$
V(t, x):=\inf \left\{g\left(y^{u}(T)\right) \mid y^{u}(\cdot) \text { solves }(1) \text { on }[t, T] \text { with } u(\cdot) \in \mathcal{U}, y^{u}(t)=x\right\} \text {. }
$$

We have the following result (see [31]):

Proposition 2.6. Suppose that (4) holds true and $K$ is a proximal retract set. Then the following assertions are true:

(i) (Existence of an optimal control) If $g$ is locally Lipschitz, then $V$ is locally Lipschitz. Moreover, there exist an optimal trajectory $\bar{y}^{\bar{u}}(\cdot) \in S_{F}(t, x)^{1}$ such that

$$
V(t, x)=g\left(\bar{y}^{\bar{u}}(T)\right) .
$$

(ii) (Dynamic programming principle) Let $g: K \rightarrow \mathbb{R}$ be a bounded function. We have that

$$
V(t, x)=\inf _{u(\cdot) \in \mathcal{U}}
$$

for all $(t, x) \in[0, T] \times K$ and $h>0$ small enough.

The previous result says that the value function $V$ satisfies a dynamic principle. That is equivalent with saying that $V$ is nondecreasing along any

\footnotetext{
${ }^{1} S_{F}(t, x)$ denotes the set of solutions of (1) starting in $x$ at the initial time $t$.
} 
admissible trajectory and is constant along optimal trajectories. This properties are used to prove that the value function $V$ is the only solution (in viscosity sense) of the following Hamilton-Jacobi inclusion (see [31]):

$$
\left\{\begin{array}{l}
\nabla_{t} V(t, x)+\min _{u \in U}\left\langle\left(f(x, u), \nabla_{x} V(t, x)\right\rangle-\left\langle\nabla_{x} V(t, x), N_{K}(x)\right)\right\rangle \ni 0, \\
\quad \text { if }(t, x) \in[0, T) \times K, \\
\text { with the final condition } V(T, x)=g(x), \text { if } x \in K .
\end{array}\right.
$$

For a detailed treatment of this type of solution, see $[4,5,14,21-23,31]$

\section{Main results}

Firstly, we present a result characterizing optimal trajectories. Since the value function $V$ is Lipschitz continuous, we can prove that it admits some directional derivatives ${ }^{2}$ along any admissible trajectory. More precisely,

Lemma 3.1. Suppose that (4) holds true and $K$ is a proximal retract set. Then, for every trajectory $y(\cdot):=y^{u}(\cdot)$ solution of $(1)$ on $[0, T]$ and for almost every $t \in[0, T]$, there exists the directional derivative

$$
\frac{\partial V}{\partial\left(1, y^{\prime}(t)\right)}(t, y(t))
$$

This result can be obtained easily thanks to the Lipshitz property of $V$ and absolute continuity of the trajectory $y^{u}(\cdot)$.

To characterize optimal trajectories, we introduce a feedback map $G$ defined on $[0, T] \times \mathbb{R}^{N}$ by

$$
G(t, x):=\left\{v \in f(x, U)-N_{K}(x), \frac{\partial V}{\partial(1, v)}(t, x)=0\right\} .
$$

Lemma 3.2. Suppose that (4) holds true and $K$ is a proximal retract set. The following two statements are equivalent:

(i) $y(\cdot):=y^{u}(\cdot)$ is a trajectory solution of (1) on time interval $\left[t_{0}, T\right]$ and, for every $t \in\left[t_{0}, T\right], V\left(t, y^{u}(t)\right)=g\left(y^{u}(T)\right.$ ) (which means that $y^{u}(\cdot)$ is an optimal trajectory on $\left.\left[t_{0}, T\right]\right)$.

(ii) $y(\cdot)$ is a trajectory of the differential inclusion:

$$
y^{\prime}(t) \in G(t, y(t)) \quad \text { for a.e. } t \in\left[t_{0}, T\right] .
$$

Proof. Assume that (i) is satisfied, and set $\Phi(t):=V(t, y(t))$ on $\left[t_{0}, T\right]$. Since the value function $V$ is Lipschitz and since $y(\cdot)$ is absolutely continuous, we conclude that $\Phi$ is absolutely continuous and for almost every $t \in\left[t_{0}, T\right]$, we have

$$
\Phi^{\prime}(t)=\frac{\partial V}{\partial\left(1, y^{\prime}(t)\right)}(t, y(t))=0
$$

2 The directional derivative of a function $h: R^{N} \rightarrow \mathbb{R}$ at $x \in \mathbb{R}^{N}$ in the direction of $z \in \mathbb{R}^{N}$ (whenever it exists) is defined by

$$
\frac{\partial h}{\partial z}(x)=\lim _{\tau \rightarrow 0^{+}} \frac{h(x+\tau z)-h(x)}{\tau} .
$$


Therefore, for almost every $t \in\left[t_{0}, T\right], y^{\prime}(t) \in G(t, y(t))$. Assume next that (ii) holds true, Then $\Phi^{\prime}(t)=0$ for almost every $t \in\left[t_{0}, T\right]$. Consequently, $\Phi$ is constant and is equal to $V(T, y(T))=g(y(T))$. Then $y(\cdot)$ is optimal.

It is worth mentioning that $G$ may have empty images. Nevertheless, under assumptions (A1)-(A2), for every $t_{0} \in[0, T]$ and every $x_{0} \in K$, the differential inclusion (8) admits at least one solution satisfying $y\left(t_{0}\right)=x_{0}$.

Secondly, we present the main result of the paper. More precisely, we investigate some necessary optimality conditions which involve the costate variable. Let us recall the following definitions:

Definition 3.3. Let $K \subset \mathbb{R}^{N}$ be closed and $x \in K$. The second order normal cone to the set $K$ at $x$ is defined by:

$$
\begin{aligned}
N_{K}^{2}(x)= & \left\{(v, Y) \in \mathbb{R}^{N} \times S_{N} \mid\langle v, y-x\rangle+\frac{1}{2}(y-x) Y(y-x) \leq o\left(\|y-x\|^{2}\right)\right. \\
& \text { for all } \left.y \rightarrow_{K} x\right\} .
\end{aligned}
$$

Here, $\rightarrow_{K}$ denotes the convergence in $K$ and $S_{N}$ is the set of symmetric matrices of dimension $N \times N$.

Note that $N_{K}(x)$ and $N_{K}^{2}(x)$ are nonempty closed convex cones, which means that they are convex and for all $\lambda \geq 0$ and $v \in N_{K}(x)(\operatorname{resp} .(v, Y) \in$ $\left.N_{K}^{2}(x)\right)$ we have $\lambda v \in N_{K}(x)\left(\operatorname{resp} .(\lambda v, \lambda Y) \in N_{K}^{2}(x)\right)$.

Consider a subset $X \subset \mathbb{R}^{N}$ and a real function $h: X \rightarrow \mathbb{R}$. Assume that $h$ is Lipschitz continuous on $X$, then $h$ is differentiable almost everywhere in $X$, and when $h$ is not differentiable at some $x_{0} \in X$ then it is still possible to define its superdifferential by taking limits of the gradients of $h$ at neighbors of $x_{0}$.

Definition 3.4. Let $X \subset \mathbb{R}^{N}$ and $h: X \rightarrow \mathbb{R}$ be a Lipschitz continuous function, and let $x_{0} \in X$.

(i) The superdifferential of $h$ at $x_{0}$ is given by:

$$
\begin{aligned}
& \partial h\left(x_{0}\right) \\
& \quad:=\overline{c o}\left\{v \in \mathbb{R}^{N} \mid \exists x_{i} \rightarrow_{X} x_{0}, \text { with } h \text { differentiable at } x_{i} \text { and } \nabla \mathrm{h}\left(\mathrm{x}_{\mathrm{i}}\right) \rightarrow \mathrm{v}\right\} .
\end{aligned}
$$

(ii) If $h$ is of class $C^{1,1}$ on $X$, then we can define its second order superdifferential as:

$$
\begin{aligned}
& \partial^{2} h\left(x_{0}\right) \\
& \quad:=\overline{c o}\left\{Y \in S_{N} \mid \exists x_{i} \rightarrow_{X} x_{0}, \text { with } h \text { twice differentiable at } x_{i} \text { and } \nabla^{2} h\left(x_{i}\right) \rightarrow Y\right\} .
\end{aligned}
$$

Theorem 3.5. (i) Assume that (A1), (A2) hold and that $q:=d_{K}$ is $C^{1,1}$ and let $\left(\bar{y}^{\bar{u}}(\cdot), \bar{u}(\cdot)\right)$ be an optimal pair of the reflecting boundary control problem $(\mathcal{P})$. Then, there exists an absolutely continuous function $p(\cdot):[0, T] \rightarrow \mathbb{R}^{N}$ satisfying the adjoint system

$$
\left\{\begin{array}{l}
\text { i) }-p^{\prime}(t) \in p(t) \cdot\left(f_{x}\left(\bar{y}^{\bar{u}}(t), \bar{u}(t)\right)-\Pi_{S_{N}}\left(N_{K}^{2}\left(\bar{y}^{\bar{u}}(t)\right)\right)\right), \quad t \in[0, T] \\
\text { ii } p(T)=g_{x}\left(\bar{y}^{\bar{u}}(T)\right) .
\end{array}\right.
$$


Moreover, we have:

$\min _{u \in U}\left\langle p(t), f\left(\bar{y}^{\bar{u}}(t), u\right)\right\rangle=\left\langle p(t), f\left(\bar{y}^{\bar{u}}(t), \bar{u}(t)\right)\right\rangle$ for almost all $t \in[0, T]$.

(ii) Assume that (A1), (A2) hold and that $K$ is convex. Let $\left(\bar{y}_{n}^{\bar{u}_{n}}(\cdot), \bar{u}_{n}(\cdot)\right)$ be an optimal pair for the value function $V_{n}$ associated with $\left(\mathcal{P}_{n}\right)$ such that $\bar{y}_{n}^{\bar{u}_{n}}(0)=x_{n}, x_{n} \in K+\frac{\bar{M}}{n} B^{3}$ and with $x_{n} \rightarrow x$. Then, the sequence $\left(\bar{y}_{n}^{\bar{u}_{n}}(\cdot)\right)_{n}$ converges uniformly on $[0, T]$ to $\bar{y}^{\bar{u}}(\cdot)$ an optimal solution corresponding to the reflected problem $(\mathcal{P})$. There exist absolutely continuous functions $p_{n}:[0, T] \rightarrow \mathbb{R}^{N}$ solution to the adjoint system

$$
\left\{\begin{array}{c}
i)-p_{n}^{\prime}(t) \in p_{n}(t) \cdot\left(f_{x}\left(\bar{y}_{n}^{\bar{u}_{n}}(t), \bar{u}_{n}(t)\right)-n \partial^{2} q\left(\bar{y}_{n}^{\bar{u}_{n}}(t)\right)\right) \\
\text { for almost all } t \in[0, T] \\
\text { ii) } p_{n}(T)=g_{x}\left(\bar{y}_{n}^{\bar{u}_{n}}(T)\right) .
\end{array}\right.
$$

with

$$
\begin{aligned}
& \min _{u \in U}\left\langle p_{n}(t), f\left(\bar{y}_{n}^{\bar{u}_{n}}(t), u\right)\right\rangle=\left\langle p_{n}(t), f\left(\bar{y}_{n}^{\bar{u}_{n}}(t), \bar{u}_{n}(t)\right)\right\rangle \\
& \quad \text { for almost all } t \in[0, T] .
\end{aligned}
$$

Moreover, there exists a subsequence still denoted $p_{n}(\cdot)$, and there exist $p(\cdot)$ in $L^{2}\left([0, T], \mathbb{R}^{N}\right)$ such that $p_{n}(\cdot) \rightarrow p(\cdot)$ weakly in $L^{2}\left([0, T], \mathbb{R}^{N}\right)$ with $p(\cdot)$ satisfying $(9 \mathrm{~b})$.

The proof is postponed in Sect. 5 because we need preliminary results in order to complete it.

\section{Approximation of the reflected control problem}

In this section we study the approximation of the reflecting boundary Eq. (1) by a sequence of ordinary differential equations with Lipschitz continuous dynamics. We begin by describing proximal retract sets.

\subsection{Some properties of proximal retract sets}

We recall the following results from [28, Theorem 4.1, p. 5245]:

Proposition 4.1. Let $K \subset \mathbb{R}^{N}$ be proximal retract set. Then there exist $r, c>0$ such that the set-valued map $x \rightsquigarrow N_{K}(x) \cap r B+c x$ is monotone $e^{4}$ on $K$. If $K$ is convex then $x \rightsquigarrow N_{K}(x) \cap r B$ is monotone. Furthermore, the map $x \rightsquigarrow N_{K}(x)$ has a closed graph and there exists $\eta>0$ such that the map $x \rightarrow \Pi_{K}(x)$ is single-valued and is Lipshitz continuous on $(K+\eta B) \backslash K$.

Proposition 4.2. Assume that $K \subset \mathbb{R}^{N}$ is a proximal retract set. Then the following statements hold:

(i) The functions $q_{n}$ are of class $C^{1,1}$ on $R^{N}$ and have the same Lipschitz constant as $q(\cdot)$.

\footnotetext{
${ }^{3} \bar{M}:=\sup _{(y, u) \in K \times U}\|f(y, u)\|$.

${ }^{4}$ Recall that a set-valued map $G: K \rightarrow \mathbb{R}^{N}$ is monotone if $\left\langle y_{1}-y_{2}, x_{1}-x_{2}\right\rangle \geq 0$ for all $y_{i} \in G\left(x_{i}\right), i \in\{1,2\}$.
} 
(ii) The set-valued map $\partial^{2} q_{n}(\cdot): R^{N} \hookrightarrow S_{N}$ has nonempty, compact, convex images. Moreover, it is locally bounded and usc.

(iii) We have $\nabla q_{n}(x) \in N_{K}^{1}\left(\Pi_{K}(x)\right)$, and $\left\{\nabla q_{n}(x)\right\} \times \partial^{2} q_{n}(x) \subset N_{K}^{2}\left(\Pi_{K}(x)\right)$ for every $x \in R^{N}$.

(iv) Let $X \subset \mathbb{R}^{N}$ and $h: X \rightarrow \mathbb{R}$ be convex and of class $C^{1,1}$. Then $\partial^{2} h(\cdot)$ is positive semidefinite on $R^{N}$ u.s.c and locally bounded.

Proof. A proof of statement (i) is given in [28, Theorem 4.1] and [6]. From Section 2.1 of [20], we can deduce (ii) and (iv). Finally, we give a short proof of (iii). Let $x \in I$ be a point where $q_{n}$ is twice differentiable. By definition, we have that

$$
\begin{aligned}
q_{n}\left(y^{\prime}\right)-q_{n}(x)= & \left\langle y^{\prime}-x, \nabla q_{n}(x)\right\rangle+\frac{1}{2}\left(y^{\prime}-x\right) \nabla^{2} q_{n}(x)\left(y^{\prime}-x\right) \\
& +o\left(\left\|y^{\prime}-x\right\|^{2}\right)
\end{aligned}
$$

for all $y^{\prime} \rightarrow x$. Consider

$$
y^{\prime}=y-\Pi_{K}(x)+x \text { with } y \in K, y \rightarrow \Pi_{K}(x) .
$$

We obtain

$$
\begin{aligned}
q_{n}\left(y-\Pi_{K}(x)+x\right)-q_{n}(x)=\langle & \left.y-\Pi_{K}(x), \nabla q_{n}(x)\right\rangle \\
& +\frac{1}{2}\left(y-\Pi_{K}(x)\right) \nabla^{2} q_{n}(x)\left(y-\Pi_{K}(x)\right) \\
& +o\left(\left\|y-\Pi_{K}(x)\right\|^{2}\right)
\end{aligned}
$$

for all $y \in K, y \rightarrow \Pi_{K}(x)$. Consequently,

$$
\begin{aligned}
o\left(\left\|y-\Pi_{K}(x)\right\|^{2}\right) \geq\langle & \left.y-\Pi_{K}(x), \nabla q_{n}(x)\right\rangle \\
& +\frac{1}{2}\left(y-\Pi_{K}(x)\right) \nabla^{2} q_{n}(x)\left(y-\Pi_{K}(x)\right) \\
& +o\left(\left\|y-\Pi_{K}(x)\right\|^{2}\right)
\end{aligned}
$$

for all $y \in K, y \rightarrow \Pi_{K}(x)$, because

$$
q_{n}\left(y-\Pi_{K}(x)+x\right)-q_{n}(x) \leq 0 \quad \text { for all } y \in K
$$

So, $\nabla q_{n}(x) \in N_{K}^{1}\left(\Pi_{K}(x)\right)$, and $\nabla q_{n}(x) \times \nabla^{2} q_{n}(x) \in N_{K}^{2}\left(\Pi_{K}(x)\right)$. The conclusion follows by using the definition of $\partial^{2} q_{n}(\cdot)$.

Remark 4.3. We point out that the there following assertions are equivalent (see for instance Example 2.2 in [20]):

- $h(\cdot)$ is $C^{1,1}$ and convex on $K+\alpha B$,

- $\partial h(\cdot)$ is $C^{0,1}$ and monotone on $K+\alpha B$,

- $\partial^{2} h(\cdot)$ is positive semidefinite on $K+\alpha B$. 


\subsection{Approximating equations for the reflected control problem}

We set

$$
\bar{M}:=\sup _{(y, u) \in K \times U}\|f(y, u)\| .
$$

and we consider for every $x_{0} \in K, u \in \mathcal{U}$ and for every $n \geq 1$, the approximated equation:

$$
\begin{aligned}
& y^{\prime}(t)=f(y(t), u(t))-\nabla q_{n}(y(t)) \quad \text { for a.a. } t \in[0, T] \\
& y(0)=x_{0} .
\end{aligned}
$$

Here, we have approximated the multi-valued function $N_{K}(\cdot)$ by the function $x \longmapsto \frac{n}{2} \nabla q_{n}(x)$. In what follows, we shall denote by $F_{n}$ (for $n \geq 1$ ) the set-valued map defined by

$$
F_{n}(x):=f(x, U)-\nabla q_{n}(x) \text { for every } x \in \mathbb{R}^{N} .
$$

The following two propositions state the fact that any trajectory satisfying (1) can be approximated by a sequence of trajectories solutions of (11).

Proposition 4.4. Suppose that $K$ is a proximal retract set and (4) holds. For $n$ large enough, the set $K_{n}:=K+\frac{\bar{M}}{n} B$ is invariant ${ }^{5}$ under the set-valued map $F_{n}$.

Proof. Since $K$ is proximal retract, then for $n$ large enough, the projection function $\Pi_{k}: \mathbb{R}^{N} \rightarrow K$ is single valued. On the other hand, according to [1, Chapter 5, Proposition 1, p. 234] (see also [2]), $K_{n}$ is invariant under the set-valued map $F_{n}$, whenever

$$
\left\langle x-\Pi_{K}(x), v\right\rangle \leq 0 \quad \forall x \in \partial K_{n}, \forall v \in F_{n}(x) .
$$

Then we have just to check that (12) is satisfied. First, let us recall that $\nabla q_{n}(x)=n\left(x-\Pi_{K}(x)\right)$, for every $x \in K_{n}$ and for $n$ large enough (see [28, Theorem 4.1, p. 5245] and [27, Theorem 4.4, p. 1824]).

Therefore for every $u \in U$ and for every $x \in \partial K_{n}$, we have:

$$
\begin{aligned}
\left\langle x-\Pi_{K}(x), f(x, u)-\nabla q_{n}(x)\right\rangle & =\left\langle x-\Pi_{K}(x), f(x, u)\right\rangle-n\left\|x-\Pi_{K}(x)\right\|^{2} \\
& \leq \bar{M} d_{K}(x)-n d_{K}(x)^{2}=\frac{\bar{M}^{2}}{n}-\frac{\bar{M}^{2} n}{n^{2}} \leq 0 .
\end{aligned}
$$

We conclude that $K_{n}$ is invariant under $F_{n}$ for $n$ large enough.

Proposition 4.5. Suppose that $K$ is proximal retract set and (4) holds. Let $u:[0, \infty) \longmapsto U$ be a measurable control and let $y_{n}^{u}(\cdot)$ (for every $n \geq 1$ ) be the trajectory solution of (11a) starting from $x_{n}, x_{n} \in K+\frac{\bar{M}}{n} B$ and with $x_{n} \rightarrow x \in K$. Then the sequence $y_{n}^{u}(\cdot)$ converges uniformly to the solution $y^{u}(\cdot)$ of (1) starting from $x \in K$.

\footnotetext{
${ }^{5}$ invariant under a set-valued map $F$ if for every $x_{0} \in X$, all solutions to $\dot{x}(t) \in$ $F(x(t)), x(0)=x_{0}$ remain in $X$
} 
Proof. Indeed, for a fixed control $u(\cdot)$, we have the following

$$
\begin{aligned}
\frac{d}{d t} \frac{1}{2} & \left\|y_{n}^{u}(t)-y^{u}(t)\right\|^{2}=\left\langle f\left(y_{n}^{u}(t), u(t)\right)-f\left(y^{u}(t), u(t)\right), y_{n}^{u}(t)-y^{u}(t)\right\rangle \\
& -\left\langle\nabla q\left(y_{n}^{u_{n}}(t)\right)-n\left(y^{u}(t)\right), y_{n}^{u}(t)-y^{u}(t)\right\rangle \text { a.e. on }[0, T] .
\end{aligned}
$$

We can prove that $n\left(y^{u}(\cdot)\right)$ is in $N_{K}\left(y^{u}(\cdot)\right) \cap B(0, \bar{M})$ (see [31]) and moreover we have:

$$
\begin{aligned}
\frac{d}{d t} \frac{1}{2}\left\|y_{n}^{u}(t)-y^{u}(t)\right\|^{2}= & \left\langle f\left(y_{n}^{u}(t), u(t)\right)-f\left(y^{u}(t), u(t)\right), y_{n}^{u}(t)-y^{u}(t)\right\rangle \\
& -\left\langle\nabla q\left(y_{n}^{u_{n}}(s)\right)-n\left(y^{u}(t)\right), \Pi_{K}\left(y_{n}^{u}(t)\right)-y^{u}(t)\right\rangle \\
& -\left\langle\nabla q\left(y_{n}^{u_{n}}(s)\right)-n\left(y^{u}(t)\right), y_{n}^{u}(t)-\Pi_{K}\left(y_{n}^{u}(t)\right)\right\rangle \text { a.e. on }[0, T] .
\end{aligned}
$$

Consequently,

$$
\begin{aligned}
\frac{d}{d t} \frac{1}{2}\left\|y_{n}^{u}(t)-y^{u}(t)\right\|^{2} \leq & L_{f}\left\|y_{n}^{u}(t)-y^{u}(t)\right\|^{2} \\
& +c\left\|\Pi_{K}\left(y_{n}^{u}(t)\right)-y^{u}(t)\right\|^{2}+(1+\bar{M}) \frac{\bar{M}}{n} \\
\leq & L_{f}\left\|y_{n}^{u}(t)-y^{u}(t)\right\|^{2} \\
& +2 c\left\|y_{n}^{u}(t)-y^{u}(t)\right\|^{2}+2 c \frac{1}{n^{2}} \\
& +(1+\bar{M}) \frac{\bar{M}}{n} \quad \text { a.e. on }[0, T]
\end{aligned}
$$

where the constant $c$ is the same as in Proposition 4.1. Using the fact that $q\left(y_{n}^{u_{n}}(\cdot)\right) \in N_{K}\left(\Pi_{K}\left(y_{n}^{u}(\cdot)\right)\right), N_{K}(\cdot)$ is hypomonotone and Gronwall's inequality, we can find a constant $C_{1}>0$ such that

$$
\sup _{t \in[0, T]}\left\|y_{n}^{u}(t)-y^{u}(t)\right\|^{2} \leq C_{1}\left(\frac{1}{n}+\left\|x_{n}-x\right\|^{2}\right) \text {. }
$$

The previous estimation concludes the proof.

We consider the control problem:

$$
\text { minimize } \quad\left\{g\left(y_{n}^{u}(T)\right) \mid y_{n}^{u} \text { solves }(11) \text { on }[0, T] \text { with } u(\cdot) \in \mathcal{U}\right\} \text {. }
$$

The value function associated to this control problem is defined by:

$V_{n}\left(t_{0}, x_{0}\right):=\inf \left\{g\left(y_{n}^{u}(T)\right) \mid y_{n}^{u}\right.$ solves $(11 \mathrm{a})$ on $\left[t_{0}, T\right]$ with $\left.u(\cdot) \in \mathcal{U}, y_{n}^{u}\left(t_{0}\right)=x_{0}\right\}$.

We have the following convergence result for the optimal trajectories.

Lemma 4.6. Suppose that $K$ is proximal retract set and (4) holds. Let $\left(t_{0}, x_{0}\right)$ be given in $[0, T] \times K$. For every $n \geq 1$, we consider the optimal trajectory

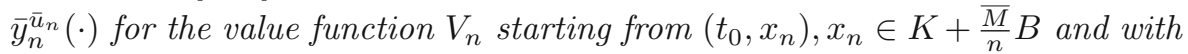
$x_{n} \rightarrow x_{0} \in K$. Then, the sequence $\left(\bar{y}_{n}^{\bar{u}_{n}}(\cdot)\right)_{n}$ converges uniformly on $\left[t_{0}, T\right]$ to $\bar{y}^{\bar{u}}(\cdot)$ an optimal solution corresponding to the problem:

$V\left(t_{0}, x_{0}\right):=\inf \left\{g\left(y^{u}(T)\right) \mid y^{u}(\cdot)\right.$ solves $(1)$ on $\left[t_{0}, T\right]$ with $\left.u(\cdot) \in \mathcal{U}, y^{u}\left(t_{0}\right)=x_{0}\right\}$ 
Proof. For $n \geq 1$, we consider $\bar{u}_{n}(\cdot)$ an optimal control associated to the optimal trajectory $\bar{y}_{n}^{\bar{u}_{n}}(\cdot)$ (see for example $[1,3,12,31]$ for results on the existence of optimal controls). Recall that the couple $\left(\bar{y}_{n}^{\bar{u}_{n}}(\cdot), \bar{u}_{n}(\cdot)\right)$ is called an optimal pair for $V_{n}$ in $\left(t_{0}, x_{n}\right)$, i. e. we have:

$$
V_{n}\left(t_{0}, x_{n}\right)=g\left(\bar{y}_{n}^{\bar{u}_{n}}(T)\right) .
$$

Note that $\left(\bar{y}_{n}^{\bar{u}_{n}}(\cdot)\right)$ converges up to a subsequence to $\bar{y}^{\bar{u}}(\cdot)$ uniformly on $\left[t_{0}, T\right]$. Indeed, we can apply Ascoli's theorem for the sequence $\left(\bar{y}_{n}^{\bar{u}_{n}}(\cdot)\right)_{n}$ because any trajectory solution of (11), starting in $\left(t_{0}, x_{n}\right)$, stays in $K_{n}:=K+\frac{\bar{M}}{n} B$ and we have:

$$
\left|f(x, u)-\nabla q_{n}(x)\right|_{\infty} \leq \bar{M}+n \frac{\bar{M}}{n}=2 \bar{M} \quad \forall x \in K_{n} .
$$

Moreover, we can employ the fact $\bar{u}_{n}(\cdot)$ has values in a compact set. Consequently, $\bar{u}_{n}(\cdot)$ converges weakly to $\bar{u}(\cdot)$ up to a subsequence. Additionally, $\left(\bar{y}^{\bar{u}}(\cdot), \bar{u}(\cdot)\right)$ is an optimal pair for $V$. Indeed on a subsequence we have

$\liminf _{n} V_{n}\left(t_{0}, x_{n}\right) \geq \lim _{k} V_{n_{k}}\left(t_{0}, x_{n_{k}}\right)=\lim _{k} g\left(\bar{y}_{n_{k}}^{\bar{u}_{n_{k}}}(T)\right)=g\left(\bar{y}^{\bar{u}}(T)\right) \geq V\left(t_{0}, x_{0}\right)$.

Moreover, for any fixed control $u(\cdot)$ we obtain the following inequalities:

$$
\limsup _{n} V_{n}\left(t_{0}, x_{n}\right) \leq \limsup _{n} g\left(y_{n}^{u}(T)\right)=\lim _{n} g\left(y_{n}^{u}(T)\right)=g\left(y^{u}(T)\right) .
$$

As consequence of the previous lemmas, we get:

Proposition 4.7. Suppose that $K$ is a compact proximal retract and (4) holds true. Then

$$
V_{n} \rightarrow V \text { pointwise on }[0, T] \times K \quad \text { whenn } \rightarrow \infty .
$$

\subsection{The adjoint systems for the approximating problems}

Problem (13) is governed by a differential equation and does not involve any state-constraint. Moreover, in this approximated control problem, the dynamics $F_{n}$ is Lipshitz while the dynamics $F$ of (3) is only usc. Hence, optimality conditions for (13) can be derived in a standard way see [12]. Let us introduce the Hamiltonian function associated to problem (13) $H: \mathbb{R}^{d} \times \mathbb{R}^{m} \times \mathbb{R}^{d} \rightarrow \mathbb{R}$ defined by $H(x, u, p):=p \cdot f(x, u)$. In the sequel, we shall adopt the convention that $p$ is a "row vector".

Theorem 4.8. Let $\left(\bar{y}_{n}^{\bar{u}_{n}}(\cdot), \bar{u}_{n}(\cdot)\right)$ be an optimal pair for the control problem (13). There exists an absolutely continuous function $p_{n}(\cdot):[0, T] \rightarrow \mathbb{R}^{N}$ solution to the adjoint system

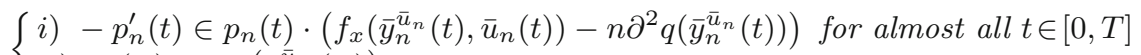
ii) $p_{n}(T)=g_{x}\left(\bar{y}_{n}^{\bar{u}_{n}}(T)\right)$.

Moreover, we have:

$\min _{u \in U}\left\langle p_{n}(t), f\left(\bar{y}_{n}^{\bar{u}_{n}}(t), u\right)\right\rangle=\left\langle p_{n}(t), f\left(\bar{y}_{n}^{\bar{u}_{n}}(t), \bar{u}_{n}(t)\right)\right\rangle$ for almost all $t \in\left[t_{0}, T\right]$. 
For the proof see for instance [12].

\section{Proof of Theorem 3.5}

The proof of (i) is a direct consequence of the regularity of the distance function, results from [12] and the fact that we have $\partial d_{K}(\cdot)=N_{K}(\cdot) \cap B($ cf. [32]). Consequently, the differential variational inequality (1):

$$
\left\{\begin{array}{l}
\text { i) } y^{\prime}(t) \in f(y(t), u(t))-N_{K}(y(t)) \quad \text { a.e } t \in[0, T] \\
i i) y(t) \in K \quad \text { for all } t \in[0, T], y(0)=x
\end{array}\right.
$$

has the same set of solutions as the unconstrained system (see [32]):

$$
\left\{\begin{array}{l}
\text { i) } y^{\prime}(t) \in f(y(t), u(t))-\bar{M} \partial d_{K}(y(t)) \quad \text { a.e } t \in[0, T] \\
\text { ii) } y(0)=x
\end{array}\right.
$$

The proof of (ii) will be split in two parts. First, we consider the sequence of adjoint state corresponding to each approximating control problem $\left(\mathcal{P}_{n}\right)$ and prove that this sequence is bounded independently on $n$ and that there exists a subsequence converging to an adjoint state of the original problem $(\mathcal{P})$. In the second part, we prove the Pontryagin principle.

In the sequel, we consider for every $n \geq 1$, an optimal pair $\left(\bar{y}_{n}^{\bar{u}_{n}}(\cdot), \bar{u}_{n}(\cdot)\right)$ solution of $\left(\mathcal{P}_{n}\right)$, and such that: $\bar{u}_{n}(\cdot) \rightarrow \bar{u}(\cdot)$ weakly in $L^{2}(0, T)$, and $\bar{y}_{n}^{\bar{u}_{n}}(\cdot) \rightarrow$ $\bar{y}(\cdot)$ in $C([0, T])$. Note that, by Lemma 4.6 , we have that $\left(\bar{y}^{\bar{u}}(\cdot), \bar{u}(\cdot)\right)$ is a solution of $(\mathcal{P})$.

Step 1. By Theorem 4.8, we know that there exists an absolutely continuous solution $p_{n}(\cdot)$ of (15a) which satisfies the following:

$$
\min _{u \in U}\left\langle p_{n}(t), f\left(\bar{y}_{n}^{\bar{u}_{n}}(t), u\right)\right\rangle=\left\langle p_{n}(t), f\left(\bar{y}_{n}^{\bar{u}_{n}}(t), \bar{u}_{n}(t)\right)\right\rangle \text { for almost all } t \in[0, T] \text {. }
$$

Indeed, (15a) has absolutely continuous solutions because $f_{x}(\cdot)$ is continuous and $\partial^{2} q_{n}(\cdot)$ has nonempty, compact, convex images and is locally bounded, u.s.c. (or closed). Moreover, we know that the map $\partial^{2} q_{n}(\cdot)$ is positive semidefinite on $K+\frac{\bar{M}}{n} B$. Thus, if we multiply by $p_{n}(\cdot)$ each member of the equality (15a), we obtain the following

$$
\left\{\begin{array}{l}
i)-\left\langle p_{n}^{\prime}(t), p_{n}(t)\right\rangle \in p_{n}(t) f_{x}\left(\bar{y}_{n}^{\bar{u}_{n}}(t), \bar{u}_{n}(t)\right) p_{n}(t)-n p_{n}(t) \partial^{2} q_{n}\left(\bar{y}_{n}^{\bar{u}_{n}}(t)\right) p_{n}(t) \\
\text { for almost all } t \in[0, T] \\
i i) p_{n}(T)=g_{x}\left(y_{n}^{\bar{u}_{n}}(T)\right) .
\end{array}\right.
$$

Consequently,

$$
\left\{\begin{array}{c}
i) \frac{d}{d t} \frac{1}{2}\left\|p_{n}(T-t)\right\|^{2} \in p_{n}(T-t) f_{x}\left(\bar{y}_{n}^{\bar{u}_{n}}(T-t), \bar{u}_{n}(T-t)\right) p_{n}(T-t) \\
\quad-n p_{n}(T-t)^{2} q_{n}\left(\bar{y}_{n}^{\bar{u}_{n}}(T-t)\right) p_{n}(T-t) \text { for almost all } t \in[0, T] \\
\text { ii }) p_{n}(T)=g_{x}\left(y_{n}^{\bar{u}_{n}}(T)\right) .
\end{array}\right.
$$

We obtain that

$$
\frac{d}{d t} \frac{1}{2}\left\|p_{n}(T-t)\right\|^{2} \leq\left(\max _{K_{n}}\left\|f_{x}\right\|\right)\left\|p_{n}(T-t)\right\|^{2} .
$$


Therefore, by Granwall Lemma, we conclude that:

$$
\left\|p_{n}(T-t)\right\|^{2} \leq e^{\left(\max _{K_{n}}\left\|f_{x}\right\|\right) 2 T}\left\|g_{x}\left(y_{n}^{\bar{u}_{n}}(T)\right)\right\|^{2}
$$

and for every $t \in[0, T]$, we have $p_{n}(t) \in e^{\max _{K_{n}}\left\|f_{x}\right\| 2 T} \max _{K_{n}}\left\|g_{x}\left(y_{n}^{\bar{u}_{n}}(\cdot)\right)\right\| B$.

We denote by $c:=\left(\max _{K_{1}}\left\|f_{x}\right\|\right) 2 T \max _{K_{1}}\left\|g_{x}(\cdot)\right\|$. Then, the family of absolutely continuous functions $\left\{p_{n}(\cdot):[0, T] \rightarrow c B\right\}$ is compact for the weak topology of the Hilbert space $L^{2}\left([0, T], \mathbb{R}^{N}\right)$. Consequently, there exists a subsequence still denoted $p_{n}(\cdot)$, and there exist $p(\cdot)$ in $L^{2}\left([0, T], \mathbb{R}^{N}\right)$ such that $p_{n}(\cdot) \rightarrow p(\cdot)$ weakly in $L^{2}\left([0, T], \mathbb{R}^{N}\right)$.

Step 2. Now, by considering that:

$$
\begin{aligned}
\int_{0}^{T} & \left\langle p_{n}(s), f\left(\bar{y}_{n}^{\bar{u}_{n}}(s), \bar{u}_{n}(s)\right)\right\rangle d s-\int_{0}^{T}\left\langle p(s), f\left(\bar{y}^{\bar{u}}(s), \bar{u}(s)\right)\right\rangle d s \\
= & \int_{0}^{T}\left\langle p_{n}(s), f\left(\bar{y}_{n}^{\bar{u}_{n}}(s), \bar{u}_{n}(s)\right)-f\left(\bar{y}^{\bar{u}}(s), \bar{u}(s)\right)\right\rangle d s \\
& +\int_{0}^{T}\left\langle p_{n}(s)-p(s), f\left(\bar{y}^{\bar{u}}(s), \bar{u}(s)\right)\right\rangle d s
\end{aligned}
$$

for every $n \geq 1$, and by using the convergence results proved in Step 1, we obtain:

$$
\lim _{n} \int_{0}^{T}\left\langle p_{n}(s), f\left(\bar{y}_{n}^{\bar{u}_{n}}(s), \bar{u}_{n}(s)\right)\right\rangle d s=\int_{0}^{T}\left\langle p(s), f\left(\bar{y}^{\bar{u}}(s), \bar{u}(s)\right)\right\rangle d s .
$$

Moreover, for every $v \in U$, we have:

$$
\lim _{n} \int_{0}^{T}\left\langle p_{n}(s), f\left(\bar{y}_{n}^{\bar{u}_{n}}(s), v\right)\right\rangle d s \geq \lim _{n} \int_{0}^{t}\left\langle p_{n}(s), f\left(\bar{y}_{n} \bar{u}_{n}(s), \bar{u}_{n}(s)\right)\right\rangle d s .
$$

Therefore, for every $v \in \mathcal{U}$, we have:

$$
\int_{0}^{T}\left\langle p(s), f\left(\bar{y}^{\bar{u}}(s), v\right)\right\rangle d s \geq \int_{0}^{T}\left\langle p(s), f\left(\bar{y}^{\bar{u}}(s), \bar{u}(s)\right)\right\rangle d s .
$$

Let $u \in U$ and $\Sigma(\bar{u}, u)$ be a subset of $[0, T]$ containing all the Lebesgue points of the functions:

$$
s \longmapsto\left\langle p(s), f\left(\bar{y}^{\bar{u}}(s), u\right)\right\rangle, \quad s \longmapsto\left\langle p(s), f\left(\bar{y}^{\bar{u}}(s), \bar{u}(s)\right)\right\rangle .
$$

Taking into account the assumptions on $f$, the Lebesgue measure of $\Sigma(\bar{u}, u)$ is equal to $T$. By considering spike perturbations of $\bar{u}(\cdot)$ by $u$, around any point $s \in \Sigma(\bar{u}, u)$, defined by:

$$
v_{k}(t):= \begin{cases}u & \text { if } t \in] s-\frac{1}{k}, s+\frac{1}{k}[ \\ \bar{u}(t) & \text { if not. }\end{cases}
$$

By passing to the limit when $k$ goes to infinity, we get that

$$
\left\langle p(s), f\left(\bar{y}^{\bar{u}}(s), u\right)\right\rangle \geq\left\langle p(s), f\left(\bar{y}^{\bar{u}}(s), \bar{u}(s)\right)\right\rangle, \quad \text { for every } s \in \Sigma(\bar{u}, u) .
$$


Let $\left(u_{k}\right)_{k \geq 1}$ be a countable dense subset of $U$. With the inequality (25), we obtain:

$$
\left\langle p(s), f\left(\bar{y}^{\bar{u}}(s), u_{k}\right)\right\rangle \geq\left\langle p(s), f\left(\bar{y}^{\bar{u}}(s), \bar{u}(s)\right)\right\rangle,
$$

for every $s \in \Sigma\left(\bar{u}, u_{k}\right)$. Now, set $\Sigma_{0}=\cap_{k \geq 1} \Sigma\left(\bar{u}, u_{k}\right)$, the Lebesgue measure of $\Sigma_{0}$ is still equal to $T$, and

$$
\left\langle p(s), f\left(\bar{y}^{\bar{u}}(s), u_{k}\right)\right\rangle \geq\left\langle p(s), f\left(\bar{y}^{\bar{u}}(s), \bar{u}(s)\right)\right\rangle
$$

for every $s \in \Sigma_{0}$ and for every $k \geq 1$. Since $f$ is continuous with respect to the control variable, we conclude that:

$$
\left\langle p(t), f\left(\bar{y}^{\bar{u}}(t), \bar{u}(t)\right)\right\rangle=\min _{u \in U}\left\langle p(t), f\left(\bar{y}^{\bar{u}}(t), u\right)\right\rangle
$$

for every $t \in \Sigma_{0}$.

Remark 5.1. Finally, we provide an example to motivate the fact that the limit $p(\cdot)$ of the sequence of absolutely continuous functions $p_{n}(\cdot)$ is nonzero. Moreover, we see that the approximated system will provide very useful hints for choosing the optimal control of the reflected system.

Indeed, we consider $K:=\left\{\left(y_{1}, y_{2}\right) \in \mathbb{R}^{2}\right.$ such that $\left.y_{2} \leq 0\right\}, U:=$ $[-1,1] \times[-1,1], f: \mathbb{R}^{2} \times U \rightarrow \mathbb{R}^{2}, f\left(y_{1}, y_{2}, u\right)=\left(u_{1}, u_{2}\right)$ and $g: \mathbb{R}^{2} \rightarrow$ $\mathbb{R}, g\left(y_{1}, y_{2}, u\right)=y_{1}+y_{2}$. Moreover, $d_{K}^{2}\left(y_{1}, y_{2}\right)=y_{2}^{2}$ if $\left(y_{1}, y_{2}\right) \notin K$ and $d_{K}^{2}\left(y_{1}, y_{2}\right)=0$ if $\left(y_{1}, y_{2}\right) \in K$. The Eq. (10a) implies that the first coordinate of $p_{n}(\cdot)=\left(p_{n_{1}}(\cdot), p_{n_{2}}(\cdot)\right)$, is nonzero. More precisely, $p_{n_{1}}(\cdot)=1$, because $p_{n 1}^{\prime}(t)=0$ a.e. $[0, T]$ and $\nabla g=(1,1)$. Consequently, $p_{1}(\cdot)=1$ a. e. $[0, T]$ and $p(\cdot) \neq 0$.

Consider $\left(x_{n 1}, x_{n 2}\right) \in K+\frac{\bar{M}}{n} B$ such that $\left(x_{n 1}, x_{n 2}\right) \rightarrow\left(x_{1}, x_{2}\right) \in K . W e$ note that $p_{n_{2}}(t)=e^{n}(t-T)$ if the optimal trajectory $\left(\bar{y}_{1}^{\bar{u}_{1}}(t), \bar{y}_{2}^{\bar{u}_{2}}\right)(t)$ starting from $\left(x_{n 1}(\cdot), x_{n 2}\right) \notin K$ is not in $K$. The limit $p(\cdot)$ is equal to $(1,0)$ a. $e$. $[0, T]$. Consequently, the relation of the form (9b) we obtain information on the first coordinate of the optimal control i. e. $\bar{u}_{1}(\cdot)=-1$. Fortunately, we are able get information on the second coordinate of the optimal control after having a look at the relation of the type $(10 \mathrm{~b})$ associated with the approximating system because $p_{n_{2}}(t)=e^{n}(t-T)$ on some time subinterval of $[0, T]$ because $\left(x_{n 1}(\cdot), x_{n 2}\right) \notin K$. Consequently, we obtain that is natural to have $\bar{u}_{2}(\cdot)=-1$ a. e. $[0, T]$.

\section{Acknowledgements}

I would like to thank F. Bonnans and H. Zidani for many fruitful discussions and for supporting my work.

\section{References}

[1] Aubin, J.P., Cellina, A.: Differential Inclusions, Set-valued Maps and Viability Theory. Springer, Berlin (1984) 
[2] Aubin, J.P., Frankowska, H.: Set-valued Analysis. Birkhäuser, Boston (1990)

[3] Bardi, M., Capuzzo-Dolcetta, I.: Optimal control and viscosity solutions of Hamilton-Jacobi-Bellman equations. In: Systems and Control: Foundations and Applications. Birkhäuser, Boston

[4] Barles, G.: Nonlinear Neumann boundary conditions for quasilinear degenerate elliptic equations and applications. J. Differ. Equ. 154(1), 191-224 (1999)

[5] Barron, E.N., Jensen, R.: Semicontinuous viscosity solutions for Hamilton-Jacobi equations with convex Hamiltonians. Commun. Partial Differ. Equ. 15(12), 1713-1742 (1990)

[6] Benoist, J.: On ergodic problem for Hamilton-Jacobi-Isaacs equations. Comptes Rendus de l'Académie Des Sciences. Série 1 Mathématique 315(8), 941$944(1992)$

[7] Bergounioux, M., Zidani, H.: Pontryagin maximum principle for optimal control of variational inequalities.. SIAM J. Control Optim. 37, 1273-1290 (1999)

[8] Bonnans, F., Tiba, D.: Control problems with mixed constraints and application to an optimal investment problem. Math. Rep. (Rom. Acad Sci.) 11(4), 293306 (2009)

[9] Bonnans, J.F., Shapiro, A.: Perturbation analysis of optimization problems. In: Springer Series in Operations Research. Springer, New York (2000)

[10] Brogliato, B., Ten Dam, A.A., Paoli, L., Génot, F., Abadie, M.: Numerical simulation of finite dimensional multibody nonsmooth mechanical systems. ASME Appl. Mech. Rev. 55(2), 107-150 (2002)

[11] Cannarsa, P., Sinestrari, C.: Semiconcave functions, Hamilton-Jacobi equations, and optimal control. In: Progress in Nonlinear Differential Equations and their Applications, vol. 58. Birkhäuser, Boston (2004)

[12] Clarke, F.: Optimization and Nonsmooth Analysis. Wiley Interscience, New York (1983)

[13] Cornet, B.: Existence of slow solutions for a class of differential inclusions. J. Math. Anal. Appl. 96, 130-147 (1983)

[14] Crandall, M.G., Ishii, H., Lions, P.L.: User's guide to viscosity solutions of second order partial differential equations. Bull. Am. Math. Soc. New Ser. 27(1), 1-67 (1992)

[15] de Pinho, M.R., Rosenblueth, J.F.: Necessary conditions for constrained problems under mangasarian-fromowitz conditions. SIAM J. Control Optim. 47(1), 535-552 (2008)

[16] Dmitruk, A.V.: Maximum principle for the general optimal control problem with phase and regular mixed constraints. Comput. Math. Model. 4(4), 364377. Software and models of systems analysis. Optimal control of dynamical systems (1993) 
[17] Frankowska, H.: A viability approach to the Skorohod problem. Stochastics 14, 227-244 (1985)

[18] Frankowska, H.: The maximum principle for an optimal solution to a differential inclusion with end points constraints. SIAM J. Control Optim. 25, 145$157(1987)$

[19] Frankowska, H., Cernea, A.: A connection between the maximum principle and dynamic programming for constrained control problems. SIAM J. Control Optim. 44(2), 673-703 (2005)

[20] Hiriart-Urruty, J.B., Strodiot, J.J., Nguyen, V.H.: Generalized hessian matrix and second-order optimality conditions for problems with $c^{1,1}$ data. Appl. Math. Optim. 11, 43-56 (1984)

[21] Lions, P.L.: Generalized Solutions of Hamilton-Jacobi Equations. Pitman Advanced Publishing Program, Boston (1982)

[22] Lions, P.L.: Neumann type boundary conditions for Hamilton-Jacobi equations. Duke Math. J. 52, 793-820 (1985)

[23] Lions, P.L., Sznitman, A.S.: Stochastic differential equations with reflecting boundary conditions. Commun. Pure Appl. Math. 37, 511-537 (1984)

[24] Milyutin, A.A., Osmolovskii, N.P.: Calculus of variations and optimal control. Translations of Mathematical Monographs. American Mathematical Society, vol 180. Providence (1998). Translated from the Russian manuscript by Dimitrii Chibisov

[25] Mordukhovich, B.S.: Variational analysis and generalized differentiation. I: Basic theory. II: Applications. Springer, Berlin (2005)

[26] Moreau, J.J.: Liaisons unilatérales sans frottement et chocs inélastiques. C. R. Acad. Sci. Paris, Sr. II 296, 1473-1476 (1938)

[27] Poliquin, R.A., Rockafellar, R.T.: Prox-regular functions in variational analysis. Trans. Am. Math. Soc. 348(5), 1805-1838 (1996)

[28] Poliquin, R.A., Rockafellar, R.T., Thibault, L.: Local differentiability of distance functions. Trans. Am. Math. Soc. 352(11), 5231-5249 (2000)

[29] Polovinkin, E.S., Smirnov, G.V.: An approach to differentiation of many-valued mapping and necessary optimality conditions for optimization of solutions of differential inclusions. Differ. Equ. 22, 660-668 (1986)

[30] Rockafellar, R.T., Wets, R.J.-B.: Variational Analysis. Springer, Berlin (1998)

[31] Serea, O.S.: On reflecting boundary problem for optimal control. SIAM J. Control Optim. 42(2), 559-575 (2003)

[32] Thibault, L.: Sweeping process with regular and nonregular sets. J. Differ. Equ. 193(1), 1-26 (2003)

[33] Vinter, R.: Optimal control. In: Modern Birkhäuser Classics. Birkhäuser, Boston (2010) 
Oana Silvia Serea

Laboratoire de Mathematiques et Physique

University of Perpignan Via Domitia

EA 4217

66860 Perpignan

France

email: oserea@yahoo.fr

Received: 8 April 2012.

Accepted: 21 October 2012. 